Reprod. Nutr. Dévelop., 1986, 26 (1 B), 245-257.

\title{
Contrôle hormonal du métabolisme hépatique chez les ruminants
}

\author{
J. GRIZARD, Michèle BALAGE, Michèle MANIN
}

Laboratoire d'Etude du Métabolisme azoté et UA CNRS 041123,

I.N.R.A., Theix, 63122 Ceyrat, France.

\section{Summary. Hormonal control of hepatic metabolism in ruminants.}

Insulin/glucagon control of hepatic metabolism, i.e. a endocrine-nervous system, is one of the general systems of integration in vertebrates. In this system, substrates coming from the digestive tract or from extrahepatic metabolism are important messenger molecules. Liver uptake of insulin and glucagon mainly accounts for high metabolic clearance rates of these hormones in both ruminants and non-ruminants. Glucagon infusion into ruminants results in an increase in the net hepatic uptake of glucose precursors and gluconeogenesis. Glucagon effects have also been demonstrated in isolated hepatocytes. Glucagon, through its effect on pyruvate carboxylase (EC 6.4.1.1.) may regulate gluconeogenesis. Insulin infusion induces hypoglycaemia. As a result, glucagon secretion increases and counterregulates insulin action. However, it has been shown that hepatic gluconeogenesis decreases during euglycaemic hyperinsulin clamp, mainly due to a decrease in the hepatic supply of glucose precursors following insulin action in extrahepatic tissues. Insulin fails to elicit any significant effect in vitro. Hepatocytes exhibit insulin and glucagon receptors. The apparent characteristics of hormone binding in vitro are similar in ruminants and non-ruminants, but the characteristics of postreceptor events are unknown in the former. Glucagon, which influences hepatic glucose synthesis, may be a major hormone in ruminants.

\section{Introduction.}

La présente revue est consacrée à l'effet des hormones sur les principales voies du métabolisme hépatique ; la néoglucogenèse est considérée en priorité puisqu'elle est essentielle pour la satisfaction des besoins en glucose des ruminants. Les mécanismes cellulaires impliqués dans le déclenchement des effets hormonaux sont aussi exposés (captage de I'hormone et interaction avec ses récepteurs, messagers et événements postrécepteurs). Les cas de l'insuline et du glucagon sont analysés en détail puisque ces deux hormones, d'une part, ont une importance considérable dans la régulation du métabolisme et, d'autre part, les connaissances sur leur mécanisme d'action ont beaucoup progressé au cours de la dernière décennie. La rareté des travaux chez les ruminants implique que les concepts généraux seront développés à partir des études réalisées chez des animaux de laboratoire, surtout le rat. Les observations effectuées chez les ruminants seront ensuite décrites avec le souci de dégager les particularités de ces 
espèces par rapport aux animaux non-ruminants. II n'y a que peu de revues qui portent sur l'étude du contrôle hormonal du métabolisme chez les ruminants (Bassett, 1975, 1978 ; Trenkle, 1978 ; Prior et Smith, 1982). De plus, aucune d'entre elles n'est spécifiquement consacrée au métabolisme hépatique.

\section{Place du contrôle hormonal du métabolisme hépatique dans les systèmes de régulation}

Le contrôle hormonal du métabolisme hépatique s'intègre dans une régulation complexe impliquant les substrats et le système nerveux et présentant de nombreuses interactions (fig. 1). En effet, les substrats (glucose, acides aminés, acides gras, minéraux) absorbés ou provenant du métabolisme extrahépatique, sont capables de modifier le métabolisme hépatique ; par exemple, la néoglucogenèse hépatique est d'autant plus forte que l'apport de substrats glucoformateurs est important. Les substrats sont aussi capables de stimuler la sécrétion d’hormones telles que celle des hormones pancréatiques (Malaisse et al., 1979 ; Sutter, 1982). Ils apportent enfin à des centres cérébraux sensibles, un message sur l'état du métabolisme périphérique (Woods et al., 1981 ; Le Magnen, 1984). Le système endocrinien, aussi bien que le système nerveux, est capable de modifier l'apport des substrats par l'aliment (régulation de l'appétit) et leur utilisation par le foie (Woods et al., 1981 ; Shimazu, 1981). Le système nerveux et le système endocrinien présentent de nombreuses relations puisque, d'une part, les glandes endocrines sont fortement dépendantes des facteurs centraux (Sutter, 1982) et, d'autre part, le système nerveux synthétise une grande variété de peptides hormonaux (revues de Yalow et Eng, 1981 ; Roth et al., 1982). Ces relations s'intègrent parfois dans un système de régulation de type "paracrine ", c'est à dire dans un système où les hormones agissent sur leur lieu de synthèse. Ceci est notamment le cas des catécholamines sécrétées dans les terminaisons nerveuses hépatiques.

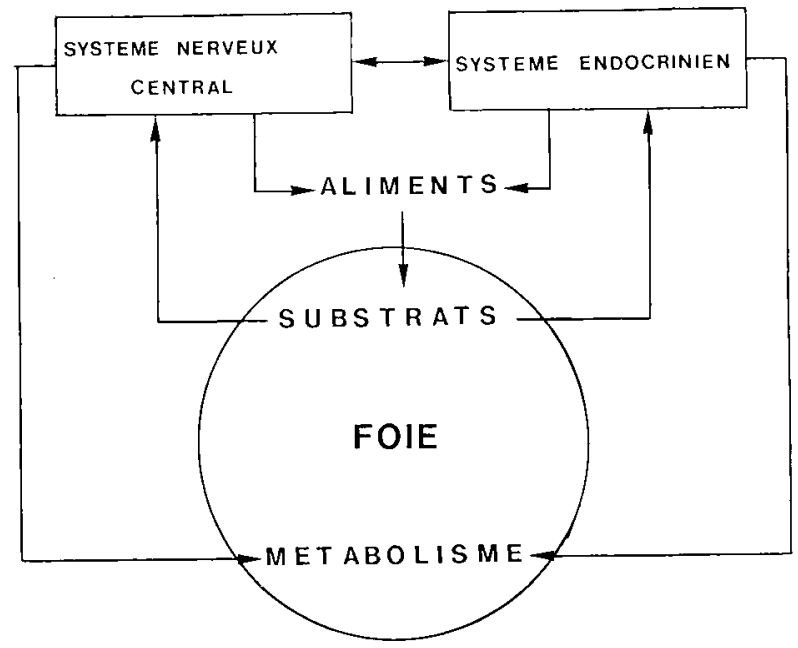

FIG. 1. - Interaction entre système nerveux central, système endocrinien et métabolisme hépatique. 


\section{Captage hépatique de l'insuline et du glucagon}

Le foie joue un rôle prépondérant dans l'élimination de l'insuline et du glucagon. II est en effet le premier organe traversé par ces hormones puisque leur sécrétion est déversée dans la veine porte via les veines gastrosplénique, gastroduodénale et mésentérique. L'étude du captage hépatique de l'insuline et du glucagon a été réalisée chez la brebis (Brockman et Bergman, 1975b ; Brockman, Manns et Bergman, 1976) et la vache (Lomax et al., 1979 (tabl. 1). Dans ces espèces, les insulinémies sont similaires à celles le plus souvent observées chez le rat $(0,5-2 \mathrm{ng} / \mathrm{ml})$. Elles sont très nettement inférieures à celles constatées après le repas chez le veau préruminant (Grizard, Patureau-Mirand et Pion, 1976 ; Grizard et al., 1982). Les taux de sécrétion d'insuline sont semblables à ceux estimés à partir des taux de clairance métabolique de l'insuline injectée par voie intrajugulaire chez le mouton $(0,8 \mu \mathrm{g} / \mathrm{kg}$, Trenkle, $1971 ; 0,6-2,3$, Grizard et Szczygiel, 1983) la chèvre (0,6-1, Grizard et al., 1985) ou les bovins (0,3-4, Geay et Grizard, 1986 ; Hart, Bines et Morand, 1980). Ils sont aussi semblables à ceux rapportés chez des espèces monogastriques comme le chien ou le singe (Kanazawa, Kuzuya et Ide, 1968 ; Ishiwata, Hetenyl et Vranic, 1969 ; Rubenstein et al., 1972 ; Ishida et al., 1983). Une forte part de l'insuline sécrétée (40-80\%) est captée par le foie. Toutefois la contribution de cet organe dans la clairance de l'insuline est plus faible que chez les monogastriques puisque le rapport d'extraction est fortement diminué (environ $10 \%$ au lieu de $40 \%$; Brockman et Bergman, 1975b ; Ishida et al., 1983).

\section{TABLEAU 1}

Captage hépatique de l'insuline et du glucagon chez des ruminants adultes non gestants et non lactants.

\begin{tabular}{|c|c|c|c|}
\hline \multirow{2}{*}{$\begin{array}{l}\text { Hormone } \\
\text { Espèce }\end{array}$} & \multicolumn{2}{|c|}{ Insuline } & \multirow{2}{*}{$\begin{array}{l}\text { Glucagon } \\
\text { Brebis }\left({ }^{3}\right)\end{array}$} \\
\hline & Vache $\left\langle{ }^{1}\right\rangle$ & Brebis $\left({ }^{2}\right)$ & \\
\hline $\begin{array}{l}\text { Insulinémie }(\mathrm{ng} / \mathrm{ml}) \\
\text { artérielle } \mathrm{A} \\
\text { porte } \mathrm{P}\end{array}$ & $\begin{array}{l}1,3 \\
1,9\end{array}$ & $\begin{array}{l}0,7 \\
1,1\end{array}$ & $\begin{array}{l}0,18 \\
0,26\end{array}$ \\
\hline $\begin{array}{l}\text { Sécrétion } S(\mu \mathrm{g} / \mathrm{h} / \mathrm{kg}) \\
\text { Captage hépatique } C(\mu \mathrm{g} / \mathrm{h} / \mathrm{kg}) \\
\text { Pourcentage d'extraction } \mathrm{E}\end{array}$ & $\begin{array}{r}0,67 \\
0,55 \\
14\end{array}$ & $\begin{array}{l}0,32 \\
0,15 \\
8\end{array}$ & $\begin{array}{l}0,10 \\
0,03 \\
7\end{array}$ \\
\hline
\end{tabular}

(1) Lomax et al., 1979 ; (2) Brockman et Bergman, 1975b ; (3) Brockman, Manns et Bergman,

Les concentrations hormonales dans les veines porte $(\mathrm{P})$ et hépatique $(\mathrm{H})$ ainsi que dans l'artère carotide (A) sont mesurées par radioimmunologie. Les débits sanguins porte $(p)$ et artériel hépatique (a) sont déterminés par la méthode à l'acide para-aminohippurique (Katz et Bergman; 1969). La sécrétion (S) est calculée par la relation: $S=p(P-A)$

et le captage hépatique (C) par la relation : $C=p(P-H)+a(A-H)$. Le taux d'extraction (E) est le rapport de I'hormone captée par le foie à l'hormone arrivée au foie et s'exprime par la relation:

$E=\frac{p(P-H)+a(A-H)}{p P+a A} \times 100$. 
Le captage hépatique de l'insuline chez les ruminants est sensible aux conditions nutritionnelles. Chez la brebis, un jeûne de 48 heures provoque comme chez le rat un accroissement du taux d'extraction hépatique ; l'hypoglycémie induit un effet similaire (Brockman et Bergman, 1975b ; Sodoyez-Goffaux et Thiry-Moris, 1980). Quelques autres observations pourraient également s'expliquer par des modifications de captage hépatique. Par exemple, la réduction de la demi-vie de l'hormone chez les bovins nourris de régimes riches en concentré traduit probablement un accroissement de l'élimination hépatique (Geay et Grizard, 1986). Inversement la réduction du taux de clairance de l'hormone chez les ovins soumis à une réduction des apports énergétiques et azotés de la ration pourrait être due à une baisse de l'élimination hépatique (Grizard et Szczygiel, 1983).

En revanche, le captage hépatique de l'insuline ne semble pas modifié par la lactation chez la vache (Lomax et al., 1979). La faiblesse de l'insulinémie des animaux en lactation s'explique par une réduction de la sécrétion de l'hormone (Lomax et al., 1979) aussi bien que par une élévation de la clairance (Grizard, Sornet et Champredon, 1986) dans des sites extra-hépatiques tels que la glande mammaire.

Les concentrations sanguines en glucagon de type pancréatique (mesuré par radioimmunologie au moyen d'anticorps équivalent à l'anticorps $30 \mathrm{~K}$ de Unger) sont beaucoup plus faibles que celles de l'insuline. Cela est en accord avec le fait que la sécrétion du glucagon est plus faible que celle de l'insuline. La demi-vie du glucagon est également plus courte (Brockman, Manns et Bergman, 1976 ; Grizard, Sornet et Champredon, 1986). Chez les non-ruminants, la part du foie dans l'élimination de l'hormone semble plus faible pour le glucagon que pour l'insuline. Cela n'est peut-être qu'apparent puisque la perte d'immunoréactivité de type glucagon pancréatique dans le foie est due essentiellement à l'élimination des composés de poids moléculaire voisin de $3,5 \mathrm{Kd}$. Les autres composés immunoréactifs (poids moléculaires d'environ 9 et $20 \mathrm{Kd}$ ) sont peu captés (Jaspan et al., 1981). Chez les ruminants, les études sont encore trop rares pour permettre de dégager les particularités du captage hépatique du glucagon (tabl. 1).

\section{Contrôle hormonal du métabolisme hépatique chez les non-ruminants}

Le contrôle hormonal du métabolisme hépatique s'excerce selon deux mécanismes distincts.

Le premier est direct puisqu'il résulte des effets des hormones sur les voies d'utilisation des substrats dans le foie (Exton, 1976 ; Mc Garry et Foster, 1976 ; Krauss-Friedmann, 1984). Ceux-ci peuvent être résumés de la manière suivante : - le transport des acides aminés dans le foie est stimulé par l'insuline, le glucagon, les catécholamines, I'hormone de croissance et l'AMP cyclique ;

- I'anabolisme protéique hépatique est stimulé par l'insuline, l'hormone de croissance et les glucocorticoïdes. Le catabolisme protéique hépatique est augmenté par le glucagon ;

- la néoglucogenèse et la glycogénolyse hépatiques sont stimulées par le glucagon et les catécholamines. L'action de ces hormones requiert les glucocorticoïdes ; en revanche, elle est inhibée par l'insuline ; 
- l'estérification des acides gras et la synthèse hépatique de lipoprotéines de très basse densité (VLDL) sont stimulées par l'insuline. La lipogenèse est inhibée par le glucagon et l'AMP cyclique ; en revanche, la cétogenèse est accrue par ces deux facteurs.

Le deuxième mécanisme par lequel les hormones contrôlent le métabolisme hépatique est indirect puisqu'il résulte des modifications de l'apport des substrats au foie à la suite de l'action des hormones dans des sites extrahépatiques (Young, 1980). Par exemple, l'insuline est capable de ralentir la néoglucogenèse hépatique à la suite de la diminution de l'apport de substrats glucoformateurs. En effet, en stimulant l'anabolisme protéique musculaire, l'insuline réduit l'apport d'acides aminés dans le foie, notamment d'alanine et de glutamine. En favorisant les dépôts adipeux, elle ralentit l'apport de glycérol et d'acides gras libres. L'insuline réduit aussi la libération périphérique d'acide lactique. En revanche, les glucocorticoïdes favorisent la néoglucogenèse hépatique puisqu'ils augmentent l'apport d'acides aminés dans le foie à la suite d'un ralentissement de l'anabolisme protéique musculaire. Ils potentialisent aussi les effets lipolytiques et glycogénolytiques des catécholamines mais diminuent la sensibilité à l'insuline.

\section{Particularités des ruminants}

Les particularités du métabolisme hépatique des ruminants sont présentés dans la revue de Rémésy et al. (1986). Ces animaux se caractérisent surtout par une néoglucogenèse intense (Basset, 1975). En effet, dans les conditions d'alimentation habituelles, il $n^{\prime} y$ a que peu de glucose absorbé puisque les glucides alimentaires sont convertis en acides gras volatils. Les besoins en glucose (qui sont très importants chez les animaux en lactation ou en gestation) sont assurés essentiellement par la néoglucogenèse hépatique. L'acide propionique absorbé est le précurseur d'environ la moitié du glucose synthétisé par le foie. Le captage est intense pour la glycine, l'alanine, la glutamine et la sérine alors qu'il est faible pour les acides aminés à chaîne ramifiée (Wolff et Bergman, 1972 ; Hume, Jacobson et Mitchell, 1972 ; Brockman et Bergman, 1975a ; Baird, Symonds et Ash, 1975 ; Bergman et Heitmann, 1978). L'alanine et la glutamine sont des précurseurs importants du glucose ; toutefois leur contribution à la néoglucogenèse reste limitée puisque seulement $10-25 \%$ du glucose sont synthétisés à partir des acides aminés (Lindsay, 1982). La part de l'acide lactique et du glycérol dans la synthèse du glucose est également modérée. Le métabolisme hépatique des ruminants se caractérise enfin par la faiblesse de la lipogenèse. Les acides gras métabolisés loxydés, transformés en corps cétoniques ou en lipoprotéines de très basse densité) proviennent essentiellement des acides gras libres plasmatiques.

a) Bilans hépatiques in vivo. - La connaissance du contrôle hormonal du métabolisme hépatique a été améliorée considérablement par l'étude des effets des hormones sur les bilans hépatiques des métabolites. Comme pour le métabolisme hormonal, les bilans hépatiques sont déterminés à partir des concentrations en métabolites dans la veine porte et les veines sus-hépatiques et des débits sanguins (Katz et Bergman, 1969).

L'influence du glucagon a été étudiée chez la brebis adulte non gestante et 
non lactante (Brockman et Bergman, 1975a ; Brockman et al., 1975a). Le glucagon est perfusé par voie intraportale au débit de $100 \mu \mathrm{g} / \mathrm{h}$. Pour éviter les décharges d'insuline qui pourraient masquer l'effet du glucagon, les animaux sont par ailleurs rendus diabétiques par l'alloxane et traités par de l'insuline exogène. Le glucagon entraîne un net accroissement de la production hépatique de glucose. Le captage de la glycine, de l'alanine, de la sérine, de la glutamine et de l'aparagine est accru. Cela correspond sans aucun doute à une stimulation de la néoglucogenèse puisque la conversion de $\mathrm{I}^{14} \mathrm{C}$ alanine en ${ }^{14} \mathrm{C}$ glucose à la suite du passage à travers le foie, est fortement augmentée. Les résultats suggèrent également une stimulation de la glycogénolyse au début du traitement (Brockman et Bergman, 1975a ; Brockman et al., 1975a).

L'effet de l'insuline sur le foie in vivo a été difficile à appréhender puisque le traitement hormonal entraîne une forte hypoglycémie qui provoque la sécrétion immédiate d'hormones à action hyperglycémiante (Bowen, 1963 ; West et Passey, 1967). La perfusion intraportale d'insuline $(50 \mu \mathrm{g} / \mathrm{h})$ entraîne chez la brebis des augmentations du captage hépatique des acides aminés. Celles-ci sont dues pour une grande part à l'élévation de la sécrétion du glucagon induite par l'hypoglycémie. En effet, elles ressemblent aux augmentations du captage constatées à la suite du traitement par le glucagon et elles ne se produisent pas chez les animaux chez qui l'hypoglycémie est supprimée par une perfusion de glucose (Brockman et al., 1975a). La synthèse du glucose a été estimée indirectement à partir du taux de renouvellement du glucose marqué administré par voie intraveineuse. Par cette technique, Brockman et al. (1975b) et Brockman (1983) ont montré sans ambiguïté que l'insuline est capable de ralentir la synthèse de glucose (qui est hépatique) chez les ovins en l'absence de variation de la glycémie. L'influence de l'insuline est d'autant plus intense que l'insulinémie est plus forte. II faut augmenter l'insulinémie à $2,5 \mathrm{ng} / \mathrm{nl}$ pour observer la demi-inhibition maximale. Le fait qu'une insulinémie deux fois plus faible permet d'obtenir le même effet chez l'homme semblerait traduire une sensibilité hépatique à l'insuline plus faible chez les ovins que les humains.

b) Hépatocytes isolés. - Les hépatocytes isolés (en survie ou en culture) n'ont été encore que peu utilisés pour l'étude des effets des hormones sur le métabolisme hépatique chez les ruminants. L'obtention de cellules ayant retenu leurs caractéristiques métaboliques pose de nombreux problèmes qui ont été discutés en détail par Pogson et al. (1984). Quelques travaux ont été réalisés chez les ovins au moyen d'hépatocytes isolés à partir du lobe caudé. La production de glucose par ces cellules est fonction de la présence des substrats glucoformateurs aussi bien que de l'état des réserves en glycogène ; elles est notammant diminuée chez les animaux en lactation chez qui les réserves de glycogène sont réduites (Gill et Hart, 1984). Le glucagon est capable de stimuler la production de glucose par ces cellules (Gill et Hart, 1984 ; Rémésy, communication personnelle). En revanche, il s'est avéré incapable de stimuler l'utilisation de l'acide ${ }^{14} \mathrm{C}$ palmitique (formation de $\mathrm{CO}_{2}$; libération de corps cétoniques). Aucun effet significatif de l'insuline sur la production de glucose n'a pu être enregistré (Gill et Hart, 1984). Ces constatations ne peuvent être interprétées qu'avec réserve puisque les études sont encore très incomplètes. Par exemple, aucune étude ne fait état de la stimu- 
lation par l'insuline et le glucagon du transport d'acide $\alpha$-amino isobutyrique dans les hépatocytes comme cela a été observé chez le rat (Fehlmann et al., 1981).

c) Enzymes. - L'influence des hormones sur le métabolisme hépatique s'explique parfois par la capacité de celles-ci à modifier l'activité des enzymes qui contrôlent les différentes réactions biochimiques. Par exemple dans le foie de rat, la transcription des gènes codant pour certaines enzymes du métabolisme du glucose (glucokinase EC 2. 7. 1. 2, phosphoénol pyruvate carboxykinase EC 4. 1. 1. 32) est contrôlée par l'insuline, les glucocorticoïdes et l'AMP cyclique qui est le messager du glucagon (Sibrowski et Seitz, 1984). Chez les ruminants, la régulation hormonale de l'expression des gènes des enzymes du métabolisme hépatique est encore inconnue et les études ne portent que sur les activités enzymatiques apparentes dans les fractions cellulaires préparées à partir d'homogénats de foie. Brockman et Manns (1974) ont enregistré, sur l'influence du glucagon, une élévation de l'activité de la pyruvate carboxylase (EC 6. 4.1.1) qui est une enzyme clé de la néoglucogenèse ; les autres enzymes qui contrôlent la néoglucogenèse, ont été inchangées (phosphoénol pyruvate carboxykinase, glucose-6-phosphatase EC 3. 1. 3. 9, hexose diphosphatase EC 3. 1. 3. 11). La pyruvate carboxylase est également la seule enzyme de la néoglucogenèse sensible au jeûne chez les ovins. A partir de ces constatations, Brockman et Manns (1974) émettent l'hypothèse que le glucagon augmente la néoglucogenèse chez les ovins principalement à la suite de la stimulation de l'activité de la pyruvate carboxylase. Ils suggèrent également que cela représente un mécanisme différent de celui constaté chez le rat chez qui l'activité phosphoénol pyruvate carboxykinase serait l'effecteur prépondérant. Des différences entre ruminants et non-ruminants dans le contrôle des enzymes de la néoglucogenèse ne sont pas surprenantes puisque il y a des différences de localisation intracellulaire de la pyruvate carboxylase et la phosphoénol pyruvate kinase entre ces espèces (Taylor, Wallace et Keech, 1971). Chez les bovins, la phosphoénol pyruvate carboxykinase est inhibée à la suite du traitement des animaux par la dexaméthazone alors que la pyruvate carboxylase et la propionyl CoA carboxylase (EC 6. 4. 1. 3) restent inchangées (Baird et Young, 1975).

\section{Mécanisme d'action des hormones dans le foie}

Les phénomènes moléculaires mis en jeu au cours du déclenchement de l'action hépatique des hormones ne sont pas encore totalement élucidés ; ils ont fait l'objet de nombreuses revues pendant la dernière décennie (Kahn, 1975 ; Insel, 1978 ; Roth et al., 1979 ; Roth, 1981 ; Valentine et Hollenberg, 1984 ; Claret et Binet, 1984). Parmi les hormones peptidiques le cas de l'insuline est vraisemblablement le plus étudié (Roth et al., 1979; Goldfine, 1981; Yip, Moule et Yeung, 1981 ; Jacobs et Cuatrecasas, 1981 ; Czech, 1984 ; Freychet, 1984 ; Gammeltoft, 1984). La première étape de l'action de l'insuline est l'interaction avec des récepteurs spécifiques situés sur la face externe de la membrane plasmique des hépatocytes. Cette interaction engendre une série d'événements biochimiques membranaires aussi bien qu'intracellulaires qui se traduisent finalement par des changements du métabolisme (fig. 2). Elle provoque rapidement l'internalisation des 
récepteurs dans un compartiment intracellulaire alimenté par ailleurs par les récepteurs nouvellement synthétisés (synthèse qui nécessite quelques heures); une partie des récepteurs intracellulaires est recyclée dans la membrane plasmique. En quelques minutes l'insuline est conduite à l'intérieur des cellules dans les structures golgiennes ou lysosomiales et catabolisée (Desbuquois et Postel-Vinay, 1980). Le transfert du message apporté par l'insuline s'effectuerait à la suite de l'autophosphorylation des récepteurs et de la phosphorylation d'autres protéines membranaires (Zick et al., 1983 ; Van Obberghen et al., 1983) ; celles-ci seraient suivies de l'activation de protéases membranaires et la libération éventuelle de messagers peptidiques, de l'activation de kinases et de phosphatases intracellulaires et de modifications dans la distribution de transporteurs. D'autres mécanismes de transfert du message apporté par l'insuline ont été également proposés ; ils dépendent de l'AMP cyclique, du GMP cyclique, de $\mathrm{Ca}^{2+}$, de $\mathrm{Mg}^{2+}$, du pH intracellulaire et de l'hyperpolarisation membranaire.

En revanche, l'AMP cyclique joue le rôle de second messager dans l'action du glucagon (Rodbell et al., 1971). Le mécanisme d'action des hormones stéroïdiennes est différent de celui des hormones peptidiques. En effet, les hormones stéroïdiennes se fixent à des récepteurs cytosoliques; le complexe hormonerécepteur est ensuite transloqué dans le noyau où les hormones agissent sur l'expression du génome (Schmidt et Litwack, 1982).

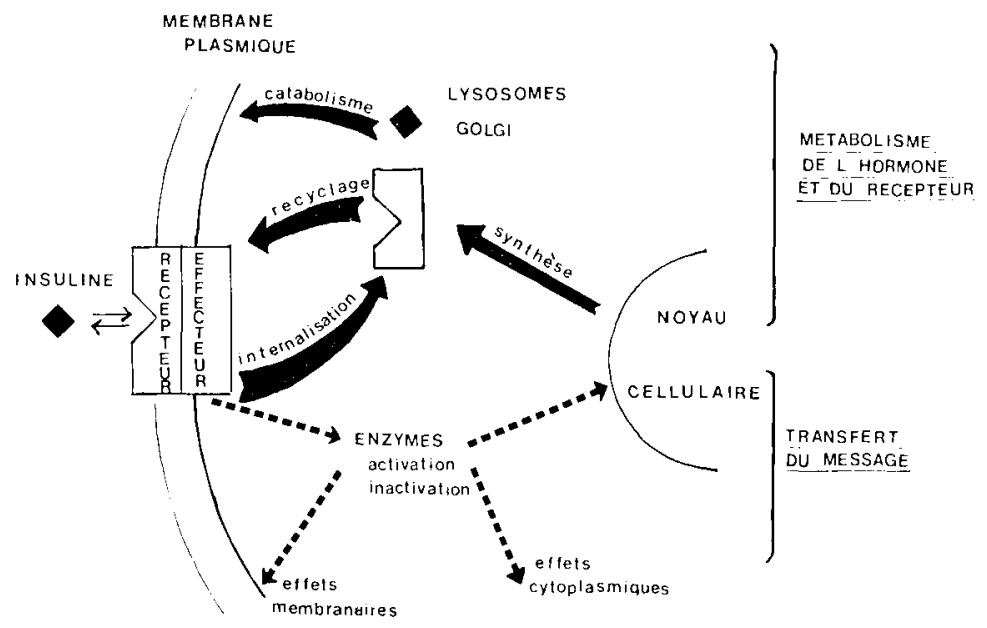

FIG. 2. - Evénements cellulaires déclenchés par l'interaction de l'insuline avec ses récepteurs.

Quelques caractéristiques seulement des récepteurs hépatiques $d$ 'insuline ont été décrites chez les ruminants à la suite de l'étude de la fixation d'insuline porcine marquée à $\left.\right|^{\prime 125} \mid$ à des préparations de microsomes (Posner et al., 1974) de membranes plasmiques (Grizard et Szczgiel, 1983) ou d'hépatocytes isolés en survie (Gill et Hart, 1980, 1981, 1982). La fixation de l'insuline à ses récepteurs est apparemment identique à celle qui est constatée chez les non-ruminants. Aux concentrations physiologiques en insuline, la plus grande part de la fixation est assurée pour chaque cellule, par quelques milliers ou dizaines de milliers de sites de 
haute affinité ( $\mathrm{Ka}$ de 0,5 à $5 \times 10^{y} \mathrm{M}^{-1}$ ). L'affinité apparente des récepteurs diminue à mesure que la concentration en insuline augmente ; cela est dû à des phénomènes de coopérativité négative entre les sites, à l'augmentation de la contribution de sites de basse affinité-haute capacité ou à d'autres phénomènes complexes. La structure subunitaire des récepteurs n'a pas encore été étudiée chez les ruminants à la différence des non-ruminants (voir revues citées en début du chapitre). Dans ces espèces, les récepteurs sont composés de 4 subunités reliées entre elles par des ponts disulfures : deux subunités $\alpha$ de poids moléculaire $135 \mathrm{Kd}$ responsables de la fixation associées à deux subunités $\beta$ de poids moléculaire $95 \mathrm{Kd}$ et porteuses d'une kinase orientée vers l'intérieur de la cellule, qui est responsable de l'autophophorylation des récepteurs. Ces subunités sont synthétisées à partir d'un seul précurseur (Hedo et al., 1983); la structure du gène de celui-ci a été déterminée (Ulrich, 1985).

La concentration en récepteurs d'insuline dans les hépatocytes de brebis augmente en fin de gestation (110-140 jours) alors que celle des récepteurs de glucagon diminue (Gill et Hart, 1982). En début de lactation (20 jours) la concentration en récepteurs d'insuline des hépatocytes est inchangée chez la brebis ; elle augmente vers le milieu de la lactation. En revanche, la concentration en récepteurs de glucagon est très faible au début de la lactation mais très forte à la mi-lactation (Gill et Hart, 1980). Chez la brebis comme chez la ratte, il n'y a pas de relation inverse entre les variations des récepteurs hépatiques d'insuline et les variations de I'insulinémie pendant le cycle gestation-lactation (Flint, 1980).

Les récepteurs hépatiques d'insuline et de glucagon chez les ruminants sont vraisemblablement sensibles aux conditions nutritionnelles de la même manière que chez les non-ruminants. La restriction énergétique provoque une augmentation de la fixation de l'insuline à ses récepteurs aussi bien chez les caprins que chez le rat alors que des variations modérées des quantités de protéines ingérées sont sans effet dans les deux espèces (Gill et Hart, 1981 ; Grizard, Arnal et Pion, 1980 ; Grizard et Szczgiel, 1983). L'augmentation des protéines ingérées stimule fortement les récepteurs de glucagon chez les caprins (Gill et Hart, 1981) ; elle est également associée à des augmentations de glucagonémie et de la capacité du glucagon à stimuler la production d'AMP cyclique chez le rat (Balage, Grizard et Rosselin, 1985).

\section{Conclusion.}

L'insuline et le glucagon participent à la régulation du métabolisme hépatique chez les ruminants aussi bien que chez les non-ruminants. Le glucagon joue un rôle majeur chez les ruminants puisqu'il stimule la néoglucogenèse hépatique, qui est essentielle pour la satisfaction des besoins en glucose dans ces espèces. En revanche, les effets de l'insuline sont vraisemblablement moins sensibles chez les ruminants que les non-ruminants. Ils s'exercent sur la néoglucogenèse hépatique surtout indirectement par la réduction de l'apport en substrats glucoformateurs dans le foie, à la suite de la stimulation de l'utilisation des substrats dans les sites extrahépatiques. Les deux hormones sont sécrétées dans la veine porte pen- 
dant les repas. L'équilibre entre les deux types de sécrétion, associé à la capacité des cellules à dégrader et répondre à chaque hormone, oriente le métabolisme hépatique. Les particularités des ruminants dans le mécanisme d'action des deux hormones, ne sont encore que peu connues.

Tères Journées sur la Nutrition et l'Alimentation des Herbivores, I.N.R.A., Paris, 21 et 22 mars 1985.

\section{Références}

BALAGE M., GRIZARD J., ROSSELIN G., 1985. Influence de régimes riches en protéines sur l'interaction glucagon-récepteur et l'activité adenyl-cyclase dans les membranes plasmiques de foie chez le rat en croissance. Réunion d'Endocrinologie de l'Association des Physiologistes, 23-24 Avril, Clermont-Ferrand.

BAIRD G. D., SYMONDS H. W., ASH R., 1975. Some observations on metabolite production and utilization in vivo by the gut and liver of adult dairy cows. J. agric. Sci. Camb., 85, 281-296.

BAIRD G. D., YOUNG J. L., 1975. The response of key gluconeogenic enzymes in bovine liver to various dietary and hormonal regimes. J. agric. Sci. Camb., 84, 227-230.

BASSET J. M., 1975. Dietary and gastrointestinal control of hormones regulating carbohydrate metabolism in ruminants, 338-398. In Mc DONALD I. W., WARNER A. C. I., Digestion and metabolism in the ruminant. Univ., New England.

BASSET J. M., 1978. Endocrine factors in the control of nutrient utilization : ruminants. Proc. Nutr. Soc., 37, 273-280.

BERGMAN E. N., HEITMANN R. N.,1978. Metabolism of amino acids by the gut, liver, kidneys, and peripheral tissues. Fed. Proc., 37, 1228-1232.

BOWEN J. M., 1963. Peripheral, hepatic and nonhepatic splanchnic effects of insulin in sheep. Cornell Vet., 54, 57-65.

BROCKMAN R. P., 1983. Effects of insulin and glucose on the production and utilization of glucose in sheep (Ovis aries). Comp. Biochem. Physiol., 74, 681-685.

BROCKMAN R. P., BERGMAN E. N., 1975a. Effect of glucagon on plasma alanine and glutamine metabolism and hepatic gluconeogenesis in sheep. Am. J. Physiol., 228, 1627-1633.

BROCKMAN R. P., BERGMAN E. N., 1975b. Quantitative aspects of insulin secretion and its hepatic and renal removal in sheep. Am. J. Physiol., 229, 1338-1343.

BROCKMAN R. P., BERGMAN E. N., JOO P. K., MANNS J. G., 1975a. Effects of glucagon and insulin on net hepatic metabolism of glucose precursors in sheep. Am. J. Physiol., 229, 13441350.

BROCKMAN R. P., BERGMAN E. N., POLLAK W. L., BRONDUM J., 1975b. Studies of glucose production in sheep using $\left(6-{ }^{3} \mathrm{H}\right)$ glucose and $\left(\mathrm{U}-{ }^{14} \mathrm{C}\right)$ glucose. Can. J. Physiol. Pharmac., 53, 1186.1189.

BROCKMAN R. P., MANNS J. G., 1974. Effects of glucagon on activities of hepatic enzymes in sheep. Cornell vet., 64, 217-224.

BROCKMAN R. P., MANNS J. G., BERGMAN E. N., 1976. Quantitative aspects of secretion and hepatic removal of glucagon in sheep. Can. J. Physiol. Pharmacol., 54, 666-670.

CLARET M., BINET A., 1984. Mécanismes d'action des hormones mobilisant le calcium dans les hépatocytes des mammifères. J. Physiol. Paris, 79, 120-128.

CZECH M. P., 1984. New perspectives on the mechanism of insulin action. Rec. Prog. Horm. Res., 40, 347-375.

DESBUQUOIS B., POSTEL-VINAY M. C., 1980. Receptors mediated internalization of insulin, glucagon, growth hormone in intact rat liver. A biochemical study, 285-292. In BRANDENBURG D., WOLLMER A., Insulin. Chemistry, structure and function of insulin and related hormones. De Gruyter W. et Co., Berlin, New York.

EXTON J. H., 1976. Hormonal control of glucogenesis, 125-167. In KLACHKO D. M., ANDERSON R. R., HEIMBERG M., Hormones and energy metabolism. Univ. Missouri, Columbia. 
FEHLMANN M., MORIN O., KITABGI P., FREYCHET $P ., 1981$. Insulin and glucagon receptors of isolated rat hepatocytes : comparison between hormone binding and amino acid transport stimulation. Endocrinology, 109, 253-261.

FLINT D. J., 1980. Changes in the number of insulin receptors of isolated rat hepatocytes during pregnancy and lactation. Biochim. biophys. Acta, 628, 322-327.

FREYCHET P., 1984. Récepteurs de l'insuline : aspects biochimiques et physiologiques. C.R. Soc. Biol., 178, 5-17.

GAMMELTOFT S., 1984. Insulin receptors: Binding kinetics and structure-function relationship of insulin. Physiol. Rev., 64, 1321-1378.

GEAY Y., GRIZARD J., 1985. Variations du métabolisme de l'insuline chez le taurillon en croissance selon la nature du régime et l'état d'engraissement. Reprod. Nutr. Dévelop., 26, 385-386.

GILL R. D., HART I. C., 1980. Properties of insulin and glucagon receptors on sheep hepatocytes : a comparison of hormone binding and plasma hormones and metabolites in lactating and non-lactating ewes. J. Endocr., 84, 237-247.

GILL R. D., HART I. C., 1981. Insulin and glucagon binding to hepatocytes in relation to circulating hormones and metabolites in goats maintained on different diets. Horm. Metab. Res., 13, 603-609.

GILL R. D., HART I. C., 1982. Hepatic receptors for insulin and glucagon in relation to plasma hormones and metabolites in pregnant and unmated ewes. J. Endocr., 93, 231-238.

GILL R. D., HART I. C., 1984. The action of various hormones and metabolites upon glucose production by isolated hepatocytes from lactating and non lactating ewes. Horm. Metab. Res., 16, 210-211.

GOLDFINE I. D., 1981. Effects of insulin on intracellular functions, 273-305. In LITWACK G., Biochemical actions of hormones, vol. 8, Acad. Press.

GRIZARD J., ARNAL M., PION R., 1980. Influence de l'hyperinsulinémie sur les récepteurs d'insuline dans le foie du rat en croissance soumis à une restriction énergétique. Reprod. Nutr. Dévelop., 20, 311-321.

GRIZARD J., PATUREAU-MIRAND P., PION R., 1976. Utilisation d'un régime riche en produits amylacés par le veau préruminant de poids élevé. II. Influence sur l'insulinémie postprandiale. Ann. Biol. anim. Bioch. Biophys., 16, 593-601.

GRIZARD J., SORNET C., BALAGE M., CHAMPREDON C., 1986. The metabolism and action of insulin and glucagon in lactating and non-lactating goats. Reprod. Nutr. Dévelop., 26 (2B) (sous presse).

GRIZARD J., SZCZYGIEL M., 1983. Insulin binding to liver plasma membranes from growing ruminating sheep maintained on different diets. Horm. Metab. Res., 15, 475-481.

GRIZARD J., TOULLEC R., GUILLOTEAU P., PATUREAU-MIRAND P., 1982. Influence de la cinétique d'évacuation gastrique de l'aliment sur l'insulinémie chez le veau préruminant. Reprod. Nutr. Dévelop., 22, 475-484.

HART I. C., BINES J. A., MORANT S. V., 1980. The secretion and metabolic clearance rates of growth hormone, insulin and prolactin in high and low-yielding cattle at four stages of lactation. Life Sci., 27, 1839-1847.

HEDO J. A., KAHN C. R., HAYASHI M., YAMADA K. M., KASUGA M., 1983. Biosynthesis and glycosylation of the insulin receptor. Evidence for a single polypeptide precursor of the two major subunits. J. biol. Chem., 258, 10020-10026.

HUME I. D., JACOBSON D. R., MITCHELL G. E., 1972. Quantitative studies on amino acid absorption in sheep. J. Nutr., 102, 495-506.

INSEL P. A., 1978. Membrane active hormones : receptors and receptor regulation, 1-42. In RICKENBERG H. V., Biochemistry and mode of action or hormones II, vol. 20, Univ. Park Press, Baltimore.

ISHIDA T., LEWIS R. M., HARTLEY C. J., ENTMAN M. L., FIELD J. B., 1983. Comparison of hepatic extraction of insulin and glucagon in conscious and anesthetized dogs. Endocrinology, 112, 1098-1109.

ISHIWATA K., HETENYL G., VRANIC M., 1969. Effect of D-glucose and D-ribose on the turnover of glucose in pancreatectomized dogs maintained on a matched intraportal infusion of insulin. Diabetes, 18, 820-827. 
JACOBS S., CUATRECASAS P., 1981. Insulin receptor: structure and function. Endocr. Rev., 2, 251-263.

JASPAN J. B., POLONSKY K. S., LEWIS M., PENSLER J., PUGH W., MOOSA A. R., RUBENSTEIN A. H., 1981. Hepatic metabolism of glucagon in the dog. Contribution of the liver to overall metabolic disposal of glucagon. Am. J. Physiol., 240, E233-E244.

KAHN C. R., 1975. Membrane receptors for polypeptide hormones, 81-146. In KORN E. D., Methods in membrane biology, vol. 3, Plenum Press, New York.

KANAZAWA Y., KUZUYA T., IDE T., 1968. Insulin output via the pancreatic vein and plasma insulin response to glucose in dogs. Am. J. Physiol., 215, 620-626.

KATZ M. L., BERGMAN E. N., 1969. Simultaneous measurements of hepatic and portal venous blood flow in the sheep and dog. Am. J. Physiol., 216, 946-952.

KRAUS-FRIEDMANN N., 1984. Hormonal regulation of hepatic gluconeogenesis. Physiol. Rev., 64, 170-259.

LARNER J., CHENG K., SCHWARTZ C., KIKUCHI K., TAMURA S., CREACY S., DUBLER R., GALASKO G., PULLIN C., KATZ M., 1982. Insulin mediators and their control of metabolism through protein phosphorylation. Rec. Prog. Horm. Res., 38, 511-556.

LE MAGNEN J., 1984. Les mécanismes de régulation du bilan d'énergie. Reprod. Nutr. Dévelop., 24, 671-692.

LINDSAY D. B., 1982. Relationships between amino acid catabolism and protein anabolism in the ruminant. Fed, Proc., 41, 2550-2554.

LOMAX M. A., BAIRD G. D., MALLINSON C. B., SYMONDS H. W., 1979. Differences between lactating and non-lactating dairy cows in concentration and secretion rate of insulin. Biochem. J., 180, 281-289.

MALAISSE W. J., SENER A., HERCHUELZ A., HUTTON J. C., 1979. Insulin release : the fuell hypothesis. Metabolism, 28, 373-386.

Mc GARRY J. D., FOSTER D. W., 1976. Hormonal control of ketogenesis, 79-96. In KLACHKO D. M., ANDERSON R. R., HEIMBERG M., Hormones and energy metabolism. Univ. Missouri, Columbia.

POGSON C. I., CARPENTER W. R., COOK J. S., FISHER M. J., LOMAX M. A., SALTER M., STANLEY J. C., 1984. A critical approach to the use of isolated liver cells for the study of metabolic events. Proc. Nutr. Soc., 43, 119-132.

POSNER B. I., KELLY P. A., SHIU P. C., FRIESEN H. G., 1974. Studies of insulin, growth hormone and prolactin binding : tissue distribution, species variation and characterization. Endocrinology, 95, 521-531.

PRIOR R. L., SMITH S. B., 1982. Hormonal effects on partitioning of nutrients for tissue growth : role of insulin. Fed. Proc., 40, 2545-2549.

RÉMÉSY C., CHILLIARD Y., RAYSSIGUIER Y., MAZUR A., DEMIGNÉ C., 1986. Le métabolisme hépatique des glucides et des lipides chez les ruminants : principales interactions durant la gestation et la lactation. Reprod. Nutr. Dévelop., 26, 205-226.

ROTH J., 1981. Insulin binding to its receptor : is the receptor more important than the hormone ? Diabetes care, 4, 27-32.

ROTH J., LE ROITH D., SHILOACH J., ROSEZWEIG J. L., LESNIAK M. A., HAVRANKOVA J., 1982. The evolutionary origins of hormones, neurotransmitters and other extracellular chemical messengers. New Engl. J. Med., 306, 523-527.

ROTH J., LESNIAK M. A., BAR R. S., MUGGEO M., MEGYESI K., HARRISON L. C., FLIER J. S., WACHSLICHT-RODARD H., GORDEN P., 1979. An introduction to receptors and receptor disorders. Proc. Soc. exp. Biol. Med., 162, 3-12.

RODBELL M., KRANS H. M. J., POHL S. L., BIRNBAUMER L., 1971. The glucagon sensitive adenyl cyclase system in plasma membranes of rat liver. J. biol. Chem., 246, 1861-1871.

RUBENSTEIN A. H., POTTENGER L. A., MAKO M., GETZ G. S., STEINER D. F., 1972. The metabolism of proinsulin and insulin by the liver. J. clin. Invest., 51, 912-921.

SCHMIDT T. J., LITWACK G., 1982. Activation of the glucocorticoid-receptor complex. Physiol. Rev., 62, 1131-1192.

SHIMAZU T., 1981. Central nervous system regulation of liver and adipose tissue metabolism. Diabetologia, 20, 343-356.

SIBROWSKI W., SEITZ H. J., 1984. Rapid action of insulin and cyclic AMP in the regulation of function messager RNA coding for glucokinase in rat liver. $J$. biol., Chem., 259, 343-346. 
SODOYEZ J. C., SODOYEZ-GOFFAUX F., THIRY-MORIS Y. M., 1980. Kinetics of insulin binding to its receptors in vivo. Effect of fasting, 293-300. In BRANDENBURG D., WOLLMER A., Insulin chemistry, structure and function of insulin and related hormones. W. De Gruyter, Berlin, New York.

SUTTER B. C. J., 1982. Regulation hormonale de la sécrétion d'insuline. J. Physiol. Paris, 78, 119130.

TAYLOR P. H., WALLACE J. D., KEECH D. B., 1971. Gluconeogenic enzymes in sheep liver. Intracellular localization of pyruvate carboxylase and phosphoenolpyruvate carboxykinase in normal, fasted and diabetic sheep. Biochim. biophys., Acta, 237, 179-191.

TRENKLE A., 1971. Postprandial changes in insulin secretion rates in sheep. J. Nutr., 101, 10991104.

TRENKLE A., 1978. Relation of hormonal variations to nutritional studies and metabolism of ruminants. J. Dairy Sci., 281-293.

ULRICH A., 1985. Insulin gene and its expression. 12th Congr. int. Diabetes Fed., Madrid.

VALENTINE K. A., HOLLENBERG M. D., 1984. Membrane receptors and hormone action, 1-51. In CANTINE M. Cell biology of the secretory process. Karger, Basel.

VAN OBBERGHEN E., ROSS! B., KOWALSKI A., GAZZANO H., PONZIO G., 1983. Receptormediated phosphorylation of the hepatic insulin receptor : evidence that the $\mathrm{Mr} 95,000$ receptor subunit is its own kinase. Proc. nat. Acad. Sci. USA, 80, 945-949.

WEST C. E., PASSEY R. F., 1967. Effect of glucose level and of insulin on the metabolism of glucose and of palmitate in sheep. Biochem. J., 102, 58-64.

WOLFF J. E., BERGMAN E. N., 1972. Gluconeogenesis from plasma amino acids in fed sheep. Am. J. Physiol., 223, 455-460.

WOLFF J. E., BERGMAN E. N., WILLIAMS H. H, 1972. Net metabolism of plasma amino acids by liver and portal-drained viscera of fed sheep. Am. J. Physiol., 223, 438-446.

WOODS S. C., WEST D. B., STEIN L. J. MCKAY L. D., LOTTER E. C., PORTE S. G., KENNEY N. J., PORTE D., 1981. Peptides and the control of meal size, Diabetologia, 20, 305-313.

YALOW R. S., ENG J., 1981. Peptide hormones in strange places. Are they there? Peptides, 2 , 17-23.

YIP C. C., MOULE M. L., YEUNG C. W. T., 1981. Characterization of the functional insulin receptor, 79-86. In MARTIN J. M., EHRLICH R. M., HOLLAND F. J., Ethiology and pathogenesis of insulin-dependent diabetes Mellitus. Raven Press, New York.

YOUNG V. R., 1980. Hormonal control of protein metabolism, with particular reference to body protein gain. In BUTTERY P. J., LINDSAY D. B., Protein-depozition in animals. Butterworths, Londion, Boston.

ZICK Y., KAZUGA M., KAHN C. R., ROTH J., 1983. Characterization of insulin-mediated phosphorylation of the insulin receptor in a cell-free system. J. biol. Chem., 258, 75-80. 